\title{
A Fuzzy Algorithm for Solving a Class of Bi-Level Linear Programming Problem
}

\author{
Lu Zhang* \\ School of Economics and Management, China University of Geosciences, Wuhan 430074, China
}

Received: 8 Aug. 2013, Revised: 10 Nov. 2013, Accepted: 11 Nov. 2013

Published online: 1 Jul. 2014

\begin{abstract}
This paper proposes a kind of bi-level linear programming problem, in which there are two decision makers in a hierarchy and they have a common variable. To deal with this bi-level problem, we introduce a virtual decision maker, who controls the common variable to maximize the sum of the objective functions of the upper and lower level decision maker (the leader and follower). To illustrate the partial cooperation, the virtual decision maker chooses his/her decision before the leader because the leader and the follower exchange the information to maximize their total benefits. Then the leader chooses his/her decision before the follower. Consequently, a tri-level programming model is obtained. Then, a fuzzy approach is presented to solve this tri-level programming. Finally, a numerical example is solved to demonstrate the feasibility of the model after presenting a fuzzy programming approach.
\end{abstract}

Keywords: Bi-level linear programming; Common variable; Tri-level programming; Fuzzy programming algorithm

\section{Introduction}

The bi-level programming is a nested optimization problem with two levels (namely the upper and lower level) in a hierarchy. It is a practical and useful tool for solving decision making (DM) problems with hierarchal structure, and has been used to solve many practical problems, such as engineering design, management, economic policy, traffic problem and so on. Therefore, the bi-level programming has been developed and researched by many authors. For the recent surveys and monographs readers can refer to $[1,2,3,4,5]$.

In a bi-level programming, the upper level decision maker (the leader) optimizes his/her objective function independently and is affected by the reaction of the lower level decision maker (the follower) who makes his/her decision after the former. Their objective functions generally conflict each other. However, most problems encountered in practice fall into the situation in which they depend partly on the degree of interaction or cooperation between them, although the information between them is incomplete and vague. So, the decision makers partially cooperate. For instance, the bi-level programming problem with a common variable is presented by considering optimal bidding strategies between the power Sellers (Power Companies) and Buyer
(Distribution Center) for contract arrangements of the middle-term contracts and the spot market transactions under uncertain electricity spot market [6]. Then, several researchers have also investigated this kind of programming problem. Lu et al. [7] proposed a comprehensive framework for bi-level multifollower programming (BLMFP) problems and Shi et al proposed an extended KuhnCTucker approach for linear BLMFP problems with shared variables among followers[8]. Later, the extended Kth-best approach[9] are also proposed for BLMFP with shared variables among followers. In the above papers, they introduce a third party called a virtual follower: the $(K+1)$ th follower controls the variable $z$, so the linear BLMFP with partial shared variables among followers is equivalently transformed into the linear BLMFP without partial shared variables among followers, which is easily solved. In this transformation, the $(K+1)$ th follower's objective function is the sum of the ith follower's objective function $(i=1,2, \cdots, K)$.

Based on the above researches, for the bi-level linear programming problem with a common variable between the leader and the follower, we introduce a third party called a virtual decision maker, who controls the the common variable, and his/her objective function is the sum of those of the leader and follower. The difference

\footnotetext{
*Corresponding author e-mail: zhanglcug@ 163.com
} 
with the above is that the virtual decision maker chooses his/her decision before the leader. Thus, a tri-level programming model is obtained, in which firstly the the virtual decision maker choose his/her decision, then the leader chooses his/her decision before the follower. Our reformation shows not only the virtual decision maker denoting the overall objectives is more important than the leader and the follower's objectives but also the leader and follower's aim to exchange the information is to maximize the sum of their objectives.

Then, we have to take the algorithm for the above model into account. The various traditional algorithms to solve the bi-level problem can be roughly classified into the following kinds[2]: extreme-point approaches for the linear case, branch-and-bound, complementary pivoting, descent methods, penalty function methods, trust-region methods and so on. However, the bi-level programming is not a convex problem, and not differentiable anywhere, and it is hard to solve. Jeroslow[10] firstly pointed out that the bi-level programming problem is a NP-Hard problem. Then, Ben-Ayed and Blair[11] and Bard[12] proved sequentially that the bi-level linear programming problem is a NP-Hard problem and searching for locally optimal solution to the bi-level linear programming is also a NP-Hard problem [13]. See Ref. [14] for more details about complexity issues about bi-level linear programming.

Recently, Shih et al [15] developed a fuzzy approach, namely interactive fuzzy decision making method, for solving the bi-level programming problems by using the concept of tolerance membership functions and multiple objective decision making. For adjusting the decision making process between the different levels and also between the decision makers of the same level, Shih and Lee [16] introduced compensatory operators. By using these compensatory operators, the solution procedures for the various types of multiple level decision problems are formulated. More interactive fuzzy decision making methods have extensively been applied to bi-level and multilevel programming problems $[17,18,19,20,21,22$, 23, 24, 25, 26,27]. While, some researchers have proposed fuzzy approaches for solving the bi-level programming problem with a common variable among the multi-followers $[28,29,30]$. In this paper, we describe a fuzzy programming approach on the basis of the above fuzzy decision making methods.

The remaining of our paper is organized as follows: Section 2 introduces the bi-level linear programming with a common variable between the leader and follower. Then, the original bi-level model is transformed into a tri-level programming model by introducing the virtual decision maker. Section 3 proposes a fuzzy programming algorithm to solve the transformed tri-level programming model. An illustrative example is provided in Section 4. Section 5 concludes this paper.

\section{Problem Formulation}

In this paper, we research the following bi-level linear programming problem

$$
\begin{gathered}
(B L P) \min _{x, y \geq 0} F_{1}(x, y, z) \\
\text { where } x, z \text { solve the following problem } \\
\min _{x, z \geq 0} F_{2}(x, y, z) \\
\text { s.t. } G(x, y, z) \leq 0
\end{gathered}
$$

where $F_{1}(x, y, z)$ and $F_{2}(x, y, z)$ are the upper and lower level objective functions, respectively. $G(x, y, z)$ is the constraint function. $y \in R^{n_{y}}$ and $z \in R^{n_{z}}$ are the decision variables controlled by the upper and lower level decision makers, respectively. $x \in R_{n_{x}}$ is the common variable between the leader and the follower. For simplicity, we denote $t=(x, y, z)$.

To transform the above BLP, we introduce a virtual decision maker, who control the common variable between the leader and follower. His/her objective function is the sum of the leader and follower's objective functions. The virtual decision maker will choose his/her decision before the original leader to illustrate his/her important status because the aim that the leader and follower change the information through the common variable is to optimize their total benefits. Therefore, we can obtain the following transformed tri-level programming model (TLP)

$(T L P) \min _{x \geq 0} F_{0}(x, y, z)=F_{1}(x, y, z)+F_{2}(x, y, z)$ (The first level) (2) where $y$ and $z$ solve the following problem

$$
\begin{aligned}
& \min _{y \geq 0} F_{1}(x, y, z) \quad \text { (The second level) } \\
& \text { where } z \text { solve the following problem } \\
& \min _{z \geq 0} F_{2}(x, y, z) \quad \text { (The third level) } \\
& \text { s.t. } G(x, y, z) \leq 0
\end{aligned}
$$

Next, some notations and definitions about tri-level programming problem are introduced:

(I)The permissible set of TLP:

$$
S=\{(x, y, z) \mid G(x, y, z) \leq 0\}
$$

(II)The projection of $S$ onto the first level decision space:

$$
S_{0}(X)=\{x \geq 0 \mid \exists(y, z), \text { such that }(x, y, z) \in S\}
$$

(III)The permissible set of the second level programming for fixed $x \in S_{0}(X)$ :

$$
S_{1}(x)=\{y \geq 0 \mid \exists z, \text { such that } G(x, y, z) \leq 0\}
$$

(IV)The rational reaction set of the second level programming for fixed $x \in S_{0}(X)$ :

$$
M_{1}(x)=\left\{y \geq 0 \mid y \in \arg \min F_{1}(x, y, z), y \in S_{1}(x)\right\}
$$


(V)The permissible set of the third level programming for fixed $x$ and $y$ :

$$
S_{2}(x, y)=\{z \geq 0 \mid G(x, y, z) \leq 0\}
$$

(VI)The rational reaction set of the third level programming for fixed $x$ and $y$ :

$$
M_{2}(x, y)=\left\{z \geq 0 \mid z \in \arg \min F_{2}(x, y, z), z \in S_{2}(x, y)\right\}
$$

(VII)The inducible region of TLP:

$$
I R=(x, y, z) \mid(x, y, z) \in S, y \in M_{1}(x), z \in M_{2}(x, y)
$$

For any tri-level programming problem, the care must be taken when the solution to the third level programming is not unique for fixed $x$ and $y$. The problem of multiple optimal solution to the third level programming can be solved by the similar method proposed to overcome the problem to bi-level programming problem[31]. Here, to ensure that the problem (2) is well posed, $S$ is assumed to be nonempty and compact, and $S_{1}(x), S_{2}(x, y), M_{1}(x)$, $M_{2}(x, y)$ are all nonempty. At the same time, we consider the situation that there is a unique solution to the third level programming for fixed $x$ and $y$. Then, the definitions of the feasibility and optimality for BLP are given as follows:

Definition $1(x, y, z) \in S$ is called the feasible solution to the problem TLP if $(x, y, z) \in I R$.

Definition $2\left(x^{*}, y^{*}, z^{*}\right) \in S$ is called the optimal solution to the problem TLP if $F_{0}\left(x^{*}, y^{*}, z^{*}\right) \leq F_{0}(x, y, z), \forall(x, y, z) \in I R$.

\section{The fuzzy programming algorithm}

To begin with a fuzzy decision making process, we obtain the optimal solution of each decision maker calculated in isolation. If the individual optimal solution $t_{i}^{*}=\left(x_{i}^{*}, y_{i}^{*}, z_{i}^{*}\right)$ are the same, then a satisfactory solution of the system has been reached.

However, they are always different because the decision makers with the conflicting objective functions behave non-cooperatively. Therefore, the fuzzy decision making process begins at the first level. To obtain a satisfactory solution, the first level decision maker should provide his/her preferred ranges for $F_{0}$ and $x$ to the second level decision maker, who has a wider feasible domain to search for his/her optimal solution.

Firstly, the membership functions are introduced by using fuzzy set theory[35]. The membership functions can be linear, piecewise linear, exponential, logarithmic, hyperbolic, inverse hyperbolic, quadratic, etc. For simplicity, we use the following linear membership function for the objective functions $F_{i}(i=0,1,2)[15]$ :

$$
\mu_{F_{i}}\left(F_{i}(t)\right)=\left\{\begin{array}{cl}
1, & F_{i}(t) \leq F_{i}^{L} \\
\frac{F_{i}^{U}-F_{i}(t)}{F_{i}^{U}-F_{i}^{L},} & F_{i}^{L} \leq F_{i}(t) \leq F_{i}^{U} \\
0, & F_{i}(t) \geq F_{i}^{U}
\end{array}\right.
$$

where $F_{i}^{U}, F_{i}^{L}$ are the upper and lower bound of $F_{i}$ on $S$. And the membership function for $x$ can be formulated as follows[20]:

$$
\mu_{x}(x)=\left\{\begin{array}{cl}
\frac{\left[x-\left(x_{1}^{*}-e_{x}^{l}\right)\right]}{e_{x}^{l}}, & x_{1}^{*}-e_{x}^{l} \leq x \leq x_{1}^{*} \\
\frac{\left[\left(x_{1}^{*}+e_{x}^{r}\right)-x\right]}{e_{x}^{r}}, & x_{1}^{*} \leq x \leq x_{1}^{*}+e_{x}^{r} \\
0, & \text { otherwise }
\end{array}\right.
$$

where the interval $\left[x_{1}^{*}-e_{x}^{l}, x_{1}^{*}+e_{x}^{r}\right]$ denotes the range of the decision of $x$ around $x_{1}^{*}$, and $e_{x}^{l}$ and $e_{x}^{r}$ are the negative and positive tolerances for $x$ at $x_{1}^{*}$, respectively.

The second level decision maker searches for the satisfactory solution to minimize his/her objective function on the basis of guaranteeing that the first level decision maker satisfies this satisfactory solution. Thus, the first level decision maker should give the minimum acceptable degree of satisfaction $\beta$ and $\alpha$ for $F_{0}$ and $x$. Hence $\mu_{F_{0}}\left(F_{0}\right) \geq \beta$ and $\mu_{x}(x) \geq \alpha$. Let $\delta$ be the minimum acceptable degree of satisfaction of the second level decision maker. So, $\mu_{F_{1}}\left(F_{1}\right) \geq \delta$. To resolve the conflict between these two decision makers and to avoid rejection of satisfactory solution by the first level decision maker, the second level decision maker must maximize $\alpha, \beta$ and $\delta$. Let $\lambda=\min \{\alpha, \beta, \delta\}$. Thus, the second level auxiliary problem is

$\max \lambda$

$$
\begin{aligned}
& \text { s.t. } \\
& \qquad(x, y, z) \leq 0 \\
& \mu_{F_{0}}\left(F_{0}\right) \geq \beta \\
& \mu_{x}(x) \geq \alpha \\
& \mu_{F_{1}}\left(F_{1}\right) \geq \delta
\end{aligned}
$$

If the two decision makers are satisfied with this solution, then the third level is also included. If not, then they may modify the tolerance values or may even change the membership functions and the second level decision maker solves a new auxiliary problem again. The process continues until the satisfactory solution is attained for top two levels after which the third level is included. Again both the higher level decision makers pass their preferred values of their decision variables and objective functions separately to the third-level decision maker. As the same as the above procedure, the third level decision maker solves the auxiliary problem as follows:

$\max \lambda$

$$
\begin{aligned}
& \text { s.t. } \\
& \qquad(x, y, z) \leq 0 \\
& \mu_{F_{0}}\left(F_{0}\right) \geq \beta \\
& \mu_{x}(x) \geq \alpha \\
& \mu_{F_{1}}\left(F_{1}\right) \geq \delta \\
& \mu_{y}(y) \geq \gamma \\
& \mu_{F_{2}}\left(F_{2}\right) \geq \varepsilon
\end{aligned}
$$

where $\gamma$ is the second level decision maker's minimum acceptable degree of satisfaction for $y . \varepsilon$ is the third level 
decision maker's minimum acceptable degree of satisfaction for $F_{2} . \lambda=\min \{\alpha, \beta, \gamma, \delta, \varepsilon\}$. And the membership function for $y$ can be formulated as follows:

$$
\mu_{y}(y)=\left\{\begin{array}{cl}
\frac{\left[y-\left(y_{2}^{*}-e_{y}^{l}\right)\right]}{e_{y}^{l}}, y_{2}^{*}-e_{y}^{l} \leq x \leq y_{2}^{*} \\
\frac{\left[\left(y_{2}^{*}+e_{y}^{r}\right)-y\right]}{e_{y}^{r}}, y_{2}^{*} \leq y \leq y_{2}^{*}+e_{y}^{r} \\
0, \quad \text { otherwise }
\end{array}\right.
$$

where the interval $\left[y_{2}^{*}-e_{y}^{l}, y_{2}^{*}+e_{y}^{r}\right]$ denotes the range of the decision of $y$ around $y_{2}^{*}$, and $e_{y}^{l}$ and $e_{y}^{r}$ are the negative and positive tolerances for $y$ at $y_{2}^{*}$, respectively.

If these three decision makers are satisfied with this solution, the overall satisfactory solution has been reached. If not, then they may modify the tolerance values or may even change the membership functions and the third level decision maker solves a new auxiliary problem again. The process continues until the satisfactory solution is attained for these three decision makers. Furthermore, the $p>3$ level programming problem can be solved by this fuzzy programming approach only by including the succeeding lower level into the system and the process repeats. The process continues until the last level is included into the system.

\section{Numerical example}

In this section, we propose a numerical example to illustrate feasibility of the proposed model.

$$
\begin{aligned}
\min _{x, y} & F_{1}(x, y, z)=-42 x_{1}+11 x_{2}+25 y_{1}-10 y_{2}-23 z_{1}-20 z_{2}(8) \\
\min _{x, z} & F_{2}(x, y, z)=-39 x_{1}+25 x_{2}+20 y_{1}+9 y_{2}-30 z_{1}-40 z_{2} \\
\text { s.t. } & -x_{1}+4 x_{2}-27 y_{1}-14 y_{2}+z_{1}-20 z_{2} \leq 1.5 \\
& 25 x_{1}-35 x_{2}-23 y_{1}+2 y_{2}+12 z_{1}+41 z_{2} \leq 13.5 \\
& 12 x_{1}+13 x_{2}-9 y_{1}-18 y_{2}+37 z_{1}-11 z_{2} \leq 5.5 \\
& -x_{1}-20 x_{2}+6 y_{1}+19 y_{2}+10 z_{1}-11 z_{2} \leq-10 \\
& 2 x_{1}+17 x_{2}-31 y_{1}-8 y_{2}-15 z_{1}-25 z_{2} \leq 4.3 \\
& -28 x_{1}-6 x_{2}+36 y_{1}-23 y_{2}+10 z_{1}-35 z_{2} \leq-5 \\
& -24 x_{1}+24 x_{2}-25 y_{1}+34 y_{2}+16 z_{1}-2 z_{2} \leq 17 \\
& 18 x_{1}+19 x_{2}+29 y_{1}-13 y_{2}-20 z_{1}+7 z_{2} \leq 45 \\
& 27 x_{1}-29 x_{2}+13 y_{1}+10 y_{2}-29 z_{1}-38 z_{2} \leq-48 \\
& x_{i} \geq 0, y_{i} \geq 0, z_{i} \geq 0, i=1,2 .
\end{aligned}
$$

where $x=\left(x_{1}, x_{2}\right)$ is the common variable. We introduce a virtual decision maker, who controls the common variable. Then the above model can be transformed into the following tri-level programming problem:

$$
\begin{aligned}
\min _{x} & F_{0}(x, y, z)=-81 x_{1}+36 x_{2}+45 y_{1}-y_{2}-53 z_{1}-60 z_{2} \\
\min _{y} & F_{1}(x, y, z)=-42 x_{1}+11 x_{2}+25 y_{1}-10 y_{2}-23 z_{1}-20 z_{2} \\
\min _{z} & F_{2}(x, y, z)=-39 x_{1}+25 x_{2}+20 y_{1}+9 y_{2}-30 z_{1}-40 z_{2} \\
\text { s.t. } & -x_{1}+4 x_{2}-27 y_{1}-14 y_{2}+z_{1}-20 z_{2} \leq 1.5 \\
& 25 x_{1}-35 x_{2}-23 y_{1}+2 y_{2}+12 z_{1}+41 z_{2} \leq 13.5 \\
& 12 x_{1}+13 x_{2}-9 y_{1}-18 y_{2}+37 z_{1}-11 z_{2} \leq 5.5 \\
& -x_{1}-20 x_{2}+6 y_{1}+19 y_{2}+10 z_{1}-11 z_{2} \leq-10 \\
& 2 x_{1}+17 x_{2}-31 y_{1}-8 y_{2}-15 z_{1}-25 z_{2} \leq 4.3 \\
& -28 x_{1}-6 x_{2}+36 y_{1}-23 y_{2}+10 z_{1}-35 z_{2} \leq-5 \\
& -24 x_{1}+24 x_{2}-25 y_{1}+34 y_{2}+16 z_{1}-2 z_{2} \leq 17 \\
& 18 x_{1}+19 x_{2}+29 y_{1}-13 y_{2}-20 z_{1}+7 z_{2} \leq 45 \\
& 27 x_{1}-29 x_{2}+13 y_{1}+10 y_{2}-29 z_{1}-38 z_{2} \leq-48 \\
& x_{i} \geq 0, y_{i} \geq 0, z_{i} \geq 0, i=1,2 .
\end{aligned}
$$

We obtain optimal solution of each decision maker calculated in isolation by using Lingo [36]. The results are listed as follows:

$F_{0}^{*}=-70.2317$ at

$t_{1}^{*}=(0.6722,1.0220,0.8325,0.7185,0.4484,1.0925)$.

$F_{1}^{*}=-36.9442$ at

$t_{2}^{*}=(0.7486,1.2323,0.1969,0.3632,0.0000,1.0175)$.

$F_{2}^{*}=-35.7039$ at

$t_{3}^{*}=(0.0455,0.9576,1.3410,0.5328, \quad 0.8568,1.5945)$.

Obviously, they can not reach a satisfactory solution because the solutions of the three decision makers are not the same.

We consider the top two level decision makers. To present the membership function, the upper and lower bounds for $F_{i}(i=0,1)$ can be calculated by use of Lingo as follows: $F_{0}^{U}=-50.4810, \quad F_{0}^{L}=-70.2317$, $F_{1}^{U}=-14.7770, F_{1}^{L}=-36.9442$. The first level decides $x_{1}=0.6722$ with 0.5 (negative) and 1 (positive) tolerance and $x_{2}=1.0220$ with 0.5 (negative) and 0.3 (positive) tolerance, where these tolerances are subjectively chosen. The satisfactory solution $(0.6786,1.0396,0.7794,0.6888$, $0.4109,1.0863)$ can be obtained by solving the second level auxiliary problem using Lingo.

The two decision makers are both satisfied with the satisfactory solution, then the third level can be included. The bounds for all objective functions are calculated by using Lingo as follows: $F_{0}^{U}=-70.1142, F_{0}^{L}=$ $-70.2317 F_{1}^{U}=-35.6462, F_{1}^{L}=-36.9442$, $F_{2}^{U}=-34.4680, F_{2}^{L}=-35.7039$. The first level decides $x_{1}=0.6722$ with 0.6 (negative) and 0.4 (positive) tolerance and $x_{2}=1.0396$ with 1 (negative) and 0.5 (positive) tolerance. The second level decides $y_{1}=0.7794$ with 0.5 (negative) and 0.4 (positive) tolerance and $y_{2}=0.6888$ with 0.6 (negative) and 0.5 (positive) tolerance. The satisfactory solution $(0.6786,1.0396,0.7794,0.6888$, $0.4109,1.0863$ ) can be obtained by solving the third level auxiliary problem using Lingo. The objective function values of the leader and follower are $F_{1}=-35.6462$ and 
$F_{2}=-34.4680$ at the satisfactory solution. The optimal function values of the leader and follower are all less than those when they make the decision by themselves because the virtual decision maker who controls the common variable wants to minimize the sum of the leader and follower's benefits through the common variable.

\section{Conclusion}

In this paper, we propose a bi-level linear programming problem with a common variable between the leader and follower. The original bi-level problem is transformed into a tri-level programming problem by introducing a virtual decision maker, who decides the common variable to minimize the sum of the leader and follower's objective functions. Then, a fuzzy programming approach is described to solve the transformed problem. The feasibility of the model is illustrated by a numerical example.

\section{Acknowledgements}

The authors would like to thank the anonymous editors and reviewers for their invaluable comments on this manuscript. This research is partially funded by the Fundamental Research Funds for the Central Universities (Nos. CUG120410, 2011JBM245).

\section{References}

[1] J. F. Bard, Practical Bilevel Optimization: Algorithms and Applications, Kluwer Academic Publishers, Dordrecht, (1998).

[2] B. Colson, P. Marcotte, G. Savard, Bilevel programming: a survey., A Quarterly Journal of Operations Research, 3, 87107 (2005).

[3] S. Dempe, Foundations of Bilevel Programming, Kluwer Academic Publishers, (2002).

[4] S. Dempe, Annotated bibliography on bilevel programming and mathematical programs with equilibrium constraints, Optimization, 52, 333-359 (2003).

[5] A. Migadalas, P. M. Paradalos, P.Värbraud, Multilevel Optimization Algorithms and Applications, Kluwer Academic Publishers, (1998).

[6] Z. Wan, C. Y. Xiao, X. J. Wang, et al., Bilevel Programming Model of Optimal Bidding Strategies under the Uncertain Electricity Markets, Automation of Electric Power System, 28, 12-16 (2004).

[7] Jie Lu, Chenggen Shi, Guangquan Zhang, On bilevel multifollower decision making: General framework and solutions, Information Sciences, 176, 1607-1627 (2006).

[8] C. Shi, J. Lu, G. Zhang, H. Zhou, An extended KuhnCTucker approach for linear bilevel multifollower programming with partial shared among followers, IEEE SMC05, The Big Island, Hawaii, USA, submitted for publication.
[9] Chenggen Shi, Hong Zhou, Jie Lu et al, The Kth-best approach for linear bilevel multifollower programming with partial shared variables among followers, Applied Mathematics and Computation, 188, 1686-1698 (2007).

[10] R. Jeroslow, The polynomial hierarchy and a simple model for competitive analysis. Mathematical Programming, 32, 146-164 (1985).

[11] O. Ben-Ayed, C. Blair, Computational difficulties of bilevel linear programming. Operations Research, 38, 556-560 (1990).

[12] J. F. Bard, Some properties of the bilevel linear programming. Journal of Optimization Theory and Applications, 68, 146-164 (1991).

[13] L. Vicente, G. Savard, J. Judice, Descent approaches for quadratic bilevel programming. Journal of Optimization Theory and Applications, 81, 379-399 (1994).

[14] X. Deng, Complexity issues in bilevel linear programming. In Migdalas,A. Pardalos, P. M. and Varbrand,P.(Eds.), Multilevel Optimization: Algorithms and Applications, Kluwer Academic Publishers, Dordrecht, 149-164 (1998).

[15] H. S. Shih, Y. J. Lai and E. S. Lee, Fuzzy approach for multi-level programming problems, Computers \& Operations Research, 23, 73-91 (1996).

[16] H.-S.Shih and E. S.Lee. Compensatory fuzzy multiple level decision making,Fuzzy Sets and Systems, 114, 71-87 (2000).

[17] Y. J. Lai, Hierarchical optimization: a satisfactory solution. Fuzzy Sets and Systems, 77, 321-335 (1996).

[18] E. S. Lee and H.-S.Shih, Fuzzy and multi-level decision making: an interactive computation approach, SpringerVerlag London, (2001).

[19] E. S. Lee. Fuzzy multiple level programming, Applied Mathematics and Computation, 120, 79-90 (2001).

[20] S.Sinha, Fuzzy programming approach to multi-level programming problems, Fuzzy Sets and Systems, 136, 189202 (2003).

[21] Z. P. Wan, G. M. Wang, K. L. Hou. An interactive fuzzy decision making method for a class of bilevel programming, Book Editor(s) Ma, J; Yin, YL; Yu, J; et al., Conference 5th International Conference on Fuzzy Systems and Knowledge Discovery Location Jinan, PEOPLES R CHINA Date, 559564 (2008).

[22] S. R. Arora, R. Gupta. Interactive fuzzy goal programming approach for bilevel programming problem, European Journal of Operational Research, 194, 368-376 (2009).

[23] S. Dempe. Comment to "interactive fuzzy goal programming approach for bilevel programming problem" by S.R. Arora and R. Gupta, European Journal of Operational Research, 212, 429-431 (2011).

[24] A. Sinha. Bilevel Multi-objective Optimization problem solving using progressively interactive EMO, Evolutionary Multi-Criterion Optimization, 269-284 (2011).

[25] Y. Zheng, Z. P. Wan, G. M. Wang. A fuzzy interactive method for a class of bilevel multiobjective programming problem, Expert Systems with Applications, 38, 1038410388 (2011).

[26] H. Katagiri, T. Uno, K. Kato, et al. Random fuzzy bilevel linear programming through possibility-based value at risk model, International Journal of Machine Learning and Cybernetics, 1-14 (2012).

[27] M. Sakawa, H. Katagiri, T. Matsui. Stackelberg solutions for fuzzy random bilevel linear programming through level 
sets and probability maximization, Operational Research, 12, 271-286 (2012)

[28] G. M. Wang, X. J. Wang, Z. P. Wan. A fuzzy interactive decision making algorithm for bilevel multi-followers programming with partial shared variables among followers, Expert Systems with Applications, 36, 10471-10474 (2009).

[29] G. Zhang, G. Zhang, Y. Gao, J. Lu. A fuzzy bilevel model and a PSO-based algorithm for day-ahead electricity market strategy making, Knowledge-Based and Intelligent Information and Engineering Systems, 736-744 (2009).

[30] G. Zhang, J. Lu. Fuzzy bilevel programming with multiple objectives and cooperative multiple followers, Journal of Global Optimization, 47, 403-419 (2010).

[31] W. F.Bialas, M. H.Karwan, Mathematical methods for multilevel planning, Reserch Report No. 79-2, (1979).

[32] J. F. Bard, Optimality condition for the bi-level programming problem, WP-104 University of Massachusetts, Bolton, (1983).

[33] H. P. Benson, On the structure and properties of a linear multilevel programming problem, Journal of Optimization Theory and Applications, 60, 353-373 (1989).

[34] E. Lakie, Linear Three-Level Programming Problem with The Application to Hierarchical Organizations, Addis Ababa University, (2007).

[35] H. J. Zimmermann, Fuzzy sets and its applications, Kluwer Academic Publishers, Hingham, MA, (1997).

[36] Lingo80.hlp, Lingo System Inc., (2003).

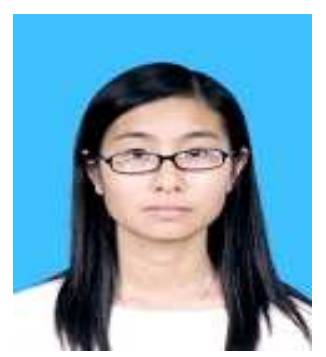

Lu Zhang is a Ph.D. candidate in Management Science and Engineering in the School of Economics and Management at China University of Geosciences (Wuhan). Funded by China Scholarship Council, she worked in Benedictine University as a visiting scholar from 2012 to 2013. Her research interests include Mathematical Economics, Organization Development, and Agricultural Economics and Management. 